\title{
La mejora de las instituciones educativas a través de las TIC
}

\author{
Educational institution improvement through ICT \\ Loreto Fernández-Cubero, Mercedes González-Sanmamed \\ Universidade da Coruña
}

\begin{abstract}
Resumen
Como parte de un trabajo de investigación más amplio, exponemos a continuación los resultados de un estudio de caso llevado a cabo en un centro educativo de la ciudad de A Coruña, en el que se está implantando el Proyecto Abalar (concreción del proyecto ministerial Escuela 2.0 y que se centra en los cursos educativos de $5^{\circ}$ y $6^{\circ}$ de Educación Primaria y $1^{\circ}$ y $2^{\circ}$ de Educación Secundaria) durante su fase inicial. De esta manera, se ha pretendido identificar los cambios que ha supuesto la incorporación al aula de las TIC, resultando determinante la implicación de la dirección y el profesorado del centro.
\end{abstract}

Palabras clave: TIC, educación primaria, proyecto Abalar, educación, innovación.

\begin{abstract}
As part of a larger research work, we present the results of a case study conducted at a school in the city of A Coruna, where Abalar project is being implemented (realization of the ministerial project Escuela 2.0 it focuses on 5th and 6th elementary education and 1st and 2nd high education) in its initial stage. This way, we have tried to identify the changes brought about by the incorporation of ICT into the classroom, both on an organizational and curricular level, being the involvement of the direction and teachers a decisive part of the project. Keywords: ICT, elementary education, Abalar project, education, innovation.
\end{abstract}

\section{Introducción}

Las tecnologías de la información y la comunicación (en adelante TIC) han modificado de forma espectacular las diferentes "formas de transmitir, clasificar $y$ procesar la información, los modos de comunicación y relación y, en consecuencia, nuestra manera de vivir y comprender el mundo actual" (González-Sanmamed, 2007, p. 219).

Este concepto, señala Rodríguez (2005), agrupa a un conjunto de técnicas, instrumentos y recursos que conforman la sociedad de la información: informática, Internet, multimedia, etc. y los sistemas de telecomunicaciones que hacen posible su distribución.

Quintana (2004) defiende un nuevo enfoque con una visión más amplia que, partiendo de la educación y de las mediaciones de la tecnología sobre ella, pone el énfasis en la educación en comunicación y en la gestión de la información.
Hay que entender que el desarrollo de las diferentes tecnologías y su incorporación a la sociedad no para de extenderse y alcanza a la mayoría de los sectores (educación, negocios, salud...): “Las TIC, hoy por hoy, forman parte de nuestras estructuras económicas, sociales y culturales. Inciden, directa o indirectamente en casi todos los aspectos de nuestra vida. Su impacto en todos los ámbitos hace cada vez más difícil prescindir de ellas" (Fernández, 2007, p. 1).

Frente a la nueva realidad que se nos presenta en la sociedad de hoy en día, la escuela no puede verse anclada en el pasado y no debe cerrarse ante las nuevas posibilidades que aquellas brindan. Como respuesta a dicha realidad "existen centros educativos que están realizando cambios para irse adaptando $e$ ir incorporando las TIC en el aula" (Domingo y Marquès, 2013).

Como reseña Cabero (2006 citado en Domingo y Marquès, 2011), las TIC configuran nuevos entornos para la formación, destacando una amplia oferta informativa, numerosas posibilidades para la orientación y tutorización, eliminación de determinadas barreras espacio-temporales, apoyo para el aprendizaje colaborativo y el autoaprendizaje, flexibilidad en el aprendizaje, etc.

Sin embargo, debemos tener en cuenta que la dotación de recursos TIC en las escuelas no es sinónimo de éxito garantizado, sino que se deben utilizar de manera correcta si realmente se pretende que ayuden en el proceso de enseñanza-aprendizaje. Pues la "presencia física de las tic en los centros no garantiza su utilización pedagógica en el aula ni el desarrollo de procesos de multialfabetización" (Area, Gross y Marzal, 2008, p. 188).

De esta integración de las TIC en el aula surge El Proyecto Abalar, una iniciativa de gran envergadura en la Comunidad Autónoma de Galicia, cofinanciada por el Fondo Europeo de Desarrollo Regional en el marco del programa operativo Feder Galicia 2007-2013. Su finalidad es conseguir la integración plena de las TIC en la práctica educativa, como una de las áreas de intervención de la Consellería de Cultura, Educación e Ordenación Universitaria que facilite un cambio en la metodología didáctica actual, mediante nuevos modelos de enseñanza que contribuyan a la modernización de la educación y a la mejora del proceso de enseñanzaaprendizaje.

El Proyecto tiene su origen en la palabra celta "Abal", manzana, la fruta de la sabiduría, y en el término gallego "Abalar", que significa mover, para transmitir el 
mensaje de promover el cambio y la transformación de la educación (Xunta de Galicia, 2015).

En el año 2012 el Proyecto contaba con la participación de 298 centros y fue galardonado con el premio "Protagonistas de la Educación 2012", para valorar el esfuerzo del Gobierno gallego en el ámbito educativo (Xunta de Galicia, 2012). Actualmente, la Red Abalar está integrada por 531 centros educativos que se extienden por toda la geografía gallega.

Como señalan Barreiro, Antelo, Mosteiro y Rodríguez (2012), Abalar nace como concreción del programa Escuela 2.0 para los centros educativos de Galicia. Pretende implicar a toda la comunidad educativa en el uso de las TIC en el aula para facilitar la búsqueda, localización, evaluación y recuperación de la información con rapidez y en diferentes formatos.

Esta integración de todos los agentes de la comunidad educativa (profesorado, alumnado, equipos directivos, familia) es prioritaria. No debemos olvidar que la estrategia de Abalar gira en torno al concepto de centro educativo digital que, superando la visión de escuela tradicional, busca disponer de recursos humanos y pedagógicos, infraestructuras y servicios adecuados para el uso cotidiano de la tecnología en la actividad docente, de aprendizajes y administrativa (Xunta de Galicia, 2015).

Por otra parte, el Proyecto Abalar persigue un salto, tanto cualitativo como cuantitativo, en el modelo educativo gallego. Pretende mejorar su eficiencia en un proceso de modernización que integra las tecnologías de la información y de la comunicación en todos los ámbitos educativos, confluyendo hacia el centro educativo digital, cada vez más preparado tecnológicamente y que busca maximizar el aprovechamiento de los recursos disponibles.

El Proyecto apuesta fundamentalmente por herramientas de software libre, que ofrecen múltiples ventajas desde el punto de vista técnico y pedagógico (Centro Nacional de Desarrollo Curricular en Sistemas no Propietarios [CeDeC], 2015).

\section{Método}

Hemos utilizado el estudio de caso para comprender e identificar los cambios que el Proyecto Abalar ha suscitado en el caso concreto de un centro educativo. Debemos comprender que, como afirma Simons (2011), la tarea fundamental de la investigación con estudio de caso es la de entender su naturaleza distintiva, pudiendo ser el caso una persona, un aula, una institución, un programa, una política o un sistema. En este estudio el caso es la escuela en sí.

Además, cabe señalar que la metodología empleada dentro de este estudio de caso se enmarca dentro de la investigación cualitativa.

\section{Participantes}

Este estudio de caso se ha realizado en un centro público de la ciudad de A Coruña dependiente de la Consellería de Cultura, Educación y Ordenación Universitaria de la Xunta de Galicia, y que abarca tanto Educación Infantil como Educación Primaria.
El centro, además, cuenta en su haber con tres líneas, y es uno de los que más alumnado acoge de la ciudad de A Coruña pues da cabida a un total de 650 alumnos y alumnas, distribuidos en 9 clases de Educación Infantil y 18 aulas de Educación Primaria.

En el CEIP Nikola Tesla (denominación ficticia para garantizar el anonimato) se ha implantado el Proyecto Abalar en el curso escolar 2011-2012, en el segundo año de vida del Proyecto, pues no le fue concedido en el año anterior, pese a haberlo solicitado en tiempo y forma, ya que la iniciativa se vinculó primeramente a los centros concertados de la ciudad y únicamente a un centro público.

En nuestro caso se ha recabado información a través del director del centro, profesional con una larga trayectoria en la enseñanza, coordinación TIC y dirección; y con amplia experiencia en el ámbito de la tecnología y la educación, y de la mano de dos docentes, ambos tutores (uno de $5^{\circ}$ de Educación Primaria y la otra de $6^{\circ}$ curso) y firmes defensores de la introducción de las TIC en el aula.

\section{Instrumentos}

Se han tenido en cuenta las recomendaciones de diversos autores sobre la variedad de técnicas e instrumentos para los diseños de investigación basados en estudios de caso (González-Sanmamed, 1994; Simons, 2011; Stake, 1998). En este estudio concreto, debemos señalar que se han realizado observaciones, entrevistas y análisis de documentos.

El contexto de observación de este estudio de caso está vinculado a una serie de clases de uno de los cursos de $6^{\circ}$ de Educación Primaria y a determinadas horas de trabajo del director y coordinador TIC del centro. En función de las dimensiones que propone Patton (1987 citado en González-Sanmamed, 1994), se trata de una observación externa, abierta, de plena información a todos los participantes, múltiples observaciones a largo plazo y de visión holística.

En cuanto al análisis documental, con el fin de comprender mejor la cultura y contexto del centro, se han analizado documentos oficiales, entre los que podemos mencionar diversa legislación educativa, documentación Abalar, la Programación General Anual del centro y el Proyecto Educativo del mismo.

En lo referente a la entrevista, hay que señalar que, puesto que se pretende conocer la implantación del Proyecto Abalar en un centro educativo concreto, se hace imprescindible abordar este conocimiento a través de los principales protagonistas e implicados en el mismo. Así, la entrevista se presenta como un instrumento ideal para conseguirlo, como señalan diversos expertos como Flick (2007), Kvale (2011) o Stake (1998).

Para este estudio de caso se ha creído conveniente la realización de entrevistas individuales a dos docentes del centro, una profesora con tutoría en $6^{\circ}$ curso de Educación Primaria y un profesor con tutoría en $5^{\circ}$, de manera que los dos cursos en los que se encuentra implantado el Proyecto Abalar queden representados.

Junto a estas entrevistas, y con el fin de tener en cuenta el punto de vista de la dirección del centro 
educativo, se ha realizado una tercera entrevista al director, que es además el coordinador TIC y coordinador Abalar, aspecto también muy relevante para la investigación. Estas entrevistas han sido realizadas en profundidad, como aconseja Simons (2011), ya que queremos conocer la perspectiva de estos agentes educativos para poder comprender mejor el proceso de implantación del Proyecto Abalar, ofreciéndoles un lienzo sobre el que plasmar su experiencia personal mediante este instrumento y a través de su propia voz. El diseño de las entrevistas se enmarca dentro del modelo que Patton (1987 citado en González-Sanmamed, 1994) denomina estructuradas abiertas: es decir, las preguntas están fijadas de antemano, pero estas permiten que los entrevistados puedan ir construyendo su propio discurso. Durante la realización de las entrevistas, los propios entrevistados reflexionan sobre el Proyecto Abalar y su implicación, sobre su experiencia y opiniones, siendo el guión previo establecido imprescindible para su desarrollo.

\section{Resultados}

En esta comunicación presentaremos los resultados relativos a la fase inicial de implementación del Proyecto Abalar en el CEIP Nikola Tesla. Concretamente, explicaremos los datos obtenidos a partir de la aplicación de los instrumentos anteriormente descritos tomando como referencia seis categorías, a través de las cuales se van comentando las visiones y acciones de cada uno de los implicados: Administración, Dirección, Profesorado, Alumnado, Familias y Recursos, en los primeros momentos de puesta en marcha de esta iniciativa.

\section{Administración}

El Proyecto Abalar ve la luz por iniciativa de la Xunta de Galicia, a través de la Consellería de Educación, Cultura e Ordenación Universitaria. Es así, que sin el apoyo de la Administración, este no podría llevarse a cabo, pues además de invertir económicamente en el Proyecto (alrededor de $12000 €$ por institución educativa), es la encargada de la concesión del mismo a los centros.

Una vez autorizada la concesión al centro sujeto de nuestro estudio, desde la Administración se dotó de los recursos tecnológicos necesarios pero hubo una falta de asesoramiento en cuanto a su uso. Además, aunque sí que es cierto que esta ofreció formación mediante una serie de cursos iniciales, estos no fueron del todo provechosos puesto que se trataba de una formación demasiado teórica y muy poco práctica como bien se señala en las entrevistas realizadas al profesorado, "se le quitó poco provecho” (EP1,4).

\section{Dirección}

En la implantación del Proyecto la figura del director se presenta como esencial, siendo clave en la gestión y organización del mismo, tomando las decisiones y liderando la idea de impulsar dicho Proyecto en el centro.
Además, este ostentaba en el momento de la implantación, y actualmente, una dualidad de cargos, director y coordinador TIC. Pero, además, hay que destacar su amplia formación en el ámbito de las tecnologías y la educación, cumpliendo así con los requisitos establecidos por la Administración en cuanto a los factores de concesión del Proyecto, como él mismo determina en la entrevista: "llevo muchos años vinculado a las nuevas tecnologías, prácticamente desde que empecé a trabajar" (ED, 2).

Cabe destacar el hecho de que el director del centro era miembro activo de la Asociación de Directores de la provincia de A Coruña, desde donde ya se habían realizado reuniones y encuentros para favorecer la implantación de las TIC en los centros.

Igualmente, el hecho de ejercer el cargo de coordinador TIC y, posteriormente, también el de coordinador Abalar viene respaldado por sus amplios conocimientos en nuevas tecnologías y su dominio de la informática del que el resto del profesorado parece ser completamente consciente, aparte de que con respecto al Proyecto Abalar era quien más convencido se mostraba.

\section{Profesorado}

En un principio, el profesorado se mostró realmente convencido de que se podría conseguir la incorporación plena de las TIC en el aula y con unas expectativas iniciales muy altas. Sin embargo, algunos docentes mostraban algunas reticencias pues había cierta incertidumbre sobre cómo iba a resultar y cómo iba a funcionar el Proyecto, "había un poco de miedo, un poco de desconocimiento de cómo iba a funcionar" (EP2, 3).

Por otro lado, debemos apuntar la existencia de distintos niveles de habilidad en cuanto al manejo de las nuevas tecnologías, buscando aquellos que se encontraban más inseguros la ayuda de sus compañeros y compañeras. Podemos deducir, a raíz de las entrevistas realizadas que, si bien la mayoría del profesorado tenía conocimientos previos sobre el Proyecto Abalar, señalan que, en mayor o menor grado, tenían que ir informándose a medida que el Proyecto se ponía en marcha. Era evidente también la necesidad de formarse adecuadamente para poder controlar los equipos, su funcionamiento y todos los aspectos que los recursos Abalar ofrecen.

\section{Alumnado}

A los alumnos y alumnas se les informó de la implantación del Proyecto Abalar una vez aceptado. Además, los estudiantes creían en un principio que el uso que se iba a dar a herramientas como los ordenadores sería a modo de juego, así como tenían unas grandes expectativas de utilizarlos durante toda la jornada lectiva, como expresa el director en su entrevista el alumnado "pensaba, inicialmente, que el uso de los ordenadores iba a ser para jugar" (ED, 9).

\section{Familias}

Desde el inicio, a las familias se les puso al corriente de las gestiones del equipo directivo de la escuela para 
su incorporación al Proyecto Abalar a través del Consejo Escolar.

Al no poder difundir toda la información a través de los representantes del Consejo, se utilizó la página web del centro como plataforma de difusión y comunicación hacia las familias. Cabe destacar aquí la cita referente a la entrevista con el director del centro, "La dirección del centro utilizó la página web del colegio para difundir a través de Internet la concesión del Proyecto" (ED, 7), página web que durante las observaciones pudo comprobarse que era atendida con asiduidad por el mismo director y coordinador TIC, "parece mostrar especial interés a la hora de mantener actualizada la página web del centro" (OB1, 4).

Cabe señalar que, previamente, a finales del curso anterior se había realizado una reunión con las familias de los estudiantes que entrarían a formar parte del Proyecto en el curso académico siguiente, explicando los equipamientos con los que se iban a contar en el aula y que su uso se daría en función de la discrecionalidad del profesorado.

\section{Recursos}

Hay que destacar que los recursos tecnológicos disponibles antes de implantarse el Proyecto Abalar eran escasos: un aula de informática equipada con 25 ordenadores de sobremesa con conexión a Internet para su empleo con cerca de 650 estudiantes.

Esto implicaba un uso muy limitado de los equipos, restringiéndose el tiempo de utilización a una o dos horas semanales.

Conjuntamente, la escuela estaba provista de un ordenador de sobremesa a disposición del profesorado y del alumnado, algunas pantallas de proyección extensibles y unos pocos proyectores que se transportaban en un carro móvil según la demanda y necesidad que se hiciese de ellos.

La idea de incorporar la tecnología al aula, potenciada por el conocimiento de algunos miembros del profesorado y la dirección del Programa Escuela 2.0 y el Modelo 1 a 1, dio pie a valorar el Proyecto Abalar como una gran oportunidad para la mejora de la institución y se pretendía, ya que a través de este Proyecto se pretendía "potencia-la utilización das novas tecnoloxías como un recurso de gran importancia nestes tempos" (DC, 4), como recoge el Proyecto Educativo del centro.

Por tanto, desde el centro se realizó una gran inversión económica $(6000 €)$ con la finalidad de acondicionar sus aulas a los requisitos que demandaba la Administración para la concesión del Proyecto, previa valoración del claustro de profesorado y aprobación del Consejo Escolar.

\section{Discusión}

A través de este estudio de caso se han podido identificar una serie de factores que han condicionado la constitución del Proyecto Abalar durante su fase inicial, destacando una serie de oportunidades, amenazas, debilidades y fortalezas que han caracterizado su implantación.

Destacan, a nivel externo, factores como los económicos, es decir, la gran inversión que se ha realizado para la dotación de recursos tecnológicos en cada aula; así como legales, referidos a como las políticas educativas autonómicas, nacionales e internacionales han contribuido a la implementación de las tecnologías en el aula; y de carácter organizativo, es decir, las excelentes relaciones con otros colegios de la ciudad o de carácter tecnológico, el avance continuo y veloz de la tecnología, recursos gratuitos para el alumnado y profesorado de $5^{\circ}$ y $6^{\circ}$ curso o la contribución a la preservación del medioambiente al apostar por la sustitución del papel.

Sin embargo, no hay que olvidar otros factores también externos como la falta de reconocimiento de la figura del coordinador TIC y la insuficiente liberación horaria o de carácter organizativo, como la carencia de una oferta de formación completa y personalizada para todos los docentes.

Ya a nivel interno, durante esta etapa inicial podríamos identificar una serie de aspectos positivos como el caso del profesorado dispuesto a aprender y a involucrase en la implementación de las TIC en el aula, el fuerte liderazgo de la dirección, la amplia formación del coordinador TIC y Abalar y su alto grado de compromiso, las infraestructuras, la motivación y disposición del alumnado suscitada por el uso de las TIC para el proceso de enseñanza-aprendizaje y el acceso personalizado y ubicuo de cada estudiante al ordenador.

Se ha de tener en cuenta que al mismo tiempo se ha tenido que hacer frente a nivel interno a una serie de factores negativos como la reticencia de una parte del profesorado a la utilización de las TIC en el aula, una escasa e inadecuada formación del profesorado en el ámbito de las TIC o la inexistencia de un plan de intervención para la evaluación interna del Proyecto y su consiguiente análisis, planificación y diseño de las oportunas líneas de mejora.

\section{Referencias}

Area, M., Gros, B. y Marzal, M. A. (2008). Alfabetizaciones y tecnologías de la información y la comunicación. Madrid: Editorial Síntesis.

Barreiro, M. J., Mosteiro, Antelo, F., Mosteiro, R. M. y Rodríguez, E. R. (2012). Proyecto Abalar: formación $\mathrm{y}$ uso de las TIC en un centro educativo digital. Organización y Gestión Educativa, 20(3), 29-32.

CeDeC. (2015). Portal web del Centro Nacional de Desarrollo Curricular en Sistemas no Propietarios. Recuperado el 15 de abril de 2015, de: http://cedec.ite.educacion.es/.

Domingo, M. y Marquès, P. (2011). Aulas 2.0 y uso de las TIC en la práctica docente. Comunicar: Revista Científica de Educomunicación, 19(37), 169-175.

Domingo, M. y Marquès, P. (2013). Práctica docente en aulas 2.0 de centros de educación primaria $\mathrm{y}$ secundaria de España. Pixel-Bit: Revista de Medios y Educación, 42, 115-128.

Fernández, M. D. (2007). ¿Contribuyen las TIC a hacer de los profesores mejores profesionales?: ¿Qué dicen los directivos escolares gallegos? Pixel-Bit: Revista de Medios y Educación, 30, p. 5-15. 
Flick, U. (2007). Introducción a la investigación cualitativa. Madrid: Morata.

González-Sanmamed, M. (1994). Aprender a enseñar: mitos y realidades. A Coruña: Universidade da Coruña, Servicio de Publicacións.

González-Sanmamed, M. (2007). Las TIC como factor de innovación y mejora de la calidad de la enseñanza. En J. Cabaro (coord.), Tecnología educativa, (pp. 219-232). Madrid: McGraw-Hill.

Kvale, S. (2011). Las entrevistas en investigación cualitativa. Madrid: Morata.

Quintana, J. (2004). La integración de las TIC en la educación primaria. Aula de Innovación Educativa, 135, 25-29.
Rodríguez, F. P. (2005). Funciones del coordinador/a TIC: estudio de caso en un centro de la capital onubense. XXI: Revista de Educación, 7, 183-192.

Simons, H. (2011). El estudio de caso: teoría y práctica. Madrid: Morata.

Stake, R. E. (1998). Investigación con estudio de casos. Madrid: Morata.

Xunta de Galicia (2012). Premio Protagonistas da Educación para o proxecto Abalar. Eduga: Revista Galega do Ensino, 64, 12.

Xunta de Galicia (2015). EspazoAbalar. Recuperado el 17 de abril de 2015, de: http://www.edu.xunta.es/espazoAbalar/es/espazo/prox ecto-abalar/introducion. 